\title{
A Review of Quantum-Dot Cellular Automata Based Adders
}

\author{
Gurmohan Singh ${ }^{1}$, R.K. Sarin ${ }^{2}$ and Balwinder Raj ${ }^{3}$ \\ Principal Engineer, Centre for Development of Advanced Computing, Mohali, \\ Punjab, India -160071 \\ ${ }^{2}$ Professor, Dr. B. R. Ambedkar National Institute of Technology, Jalandhar, \\ Punjab, India-144011 \\ ${ }^{3}$ Assistant Professor, Dr. B. R. Ambedkar National Institute of Technology, \\ Jalandhar, Punjab, India-144011 \\ 1'gurmohan@cdac.in, ${ }^{2}$ sarinrk@nitj.ac.in, ${ }^{3}$ rajb@nitj.ac.in
}

\begin{abstract}
Quantum-Dot Cellular Automata (QCA) has been emerging as a new nanocomputing paradigm. QCA computing has many advantages like very high operating speed, extremely low energy dissipation, and very high device density. An adder is heart of arithmetic units of processors. So, many researchers have been working on design and implementation of reliable and robust QCA based adder circuit. This paper presents a comprehensive review of QCA based adder designs and implementations. Being an emerging future technology having potential of replacing CMOS technology, the paper comprehensively covers an introduction to QCA nanotechnology, QCA fundamentals, QCA technology developments, evolution of QCA adders, QCA full adder designs, performance metrics, and cost function computations for coplanar and multilayer QCA 1bit full adders. The QCA adder circuits reported by various researchers have been compared in terms of adder type (coplanar or multilayer), basic full adder structure type, type and number of majority gates used, number of inverters, number of wire-crossovers, delay, and number of QCA cells. Also, the cost functions have been computed for both coplanar and multilayer QCA adders by giving higher priority to complexity.
\end{abstract}

Keywords: Quantum cellular automata (QCA), Coplanar, Multilayer, Propagation delay, Cost function, majority gate, inverter

\section{Introduction}

The continuous scaling down of feature size has pushed CMOS technology to approach its practical and theoretical limits [1]. Lot of research efforts at nanoscale are in progress to explore alternate viable technologies for future integrated circuits (ICs). QCA is emerging as a potential technology that could be used in future computing circuits/systems replacing existing Silicon technology. It provides a new computing and information transformation paradigm [2]. It is a transistor less technology that uses a square nanostructure called QCA cell comprising of 4 quantum dots [3]. Two free electrons are introduced in a four quantum dot based QCA cell which can tunnel amongst the quantum dots and take seat in any one of them. The two free electrons settle into two stable states within QCA cell that are used to encode two binary states in digital circuits. QCA cells are arranged in arrays for a particular computation and communicate with each other by Coulomb interactions. The alignment of electrons at edges of array provides the computational output. The alignment of polarizations in a QCA circuit is managed by applying an external clock and functions according to the rules of Boolean algebra [4].

This paper has been organized as follows: Section 2 presents fundamentals of QCA based computing. Section 3 discusses about QCA technology developments. Evolution of QCA adders is illustrated in Section 4. Section 5 covers QCA full adder designs and their 
comparison in detail, Section 6 illustrates performance metrics of QCA adders, and Section 7 covers cost function computations for QCA adders. Conclusions are covered in Section 8 .

\section{QCA Fundamentals}

\subsection{QCA Review}

The concept of Quantum cellular automata based digital signal processing was proposed by Lent and Tougaw [3] from Notre Dame University in 1993. The QCA cell comprises of a nanoscaled square nanostructure of 4 quantum dots. It is the most basic element used in forming arrays of cells for meaningful computations. The basic cell is depicted in Figure 1 (a). To make the QCA cell functional, two free electrons are introduced into the cell. The free electrons are allowed to tunnel within 4 quantum dots [4]. The quantum-mechanical tunneling causes electronic polarization of whole cell to settle into two stable ground states ' 1 ' and ' -1 '. These polarization states are used to encode two binary states logic ' 1 ' and ' 0 ' respectively. The binary state encoding is illustrated in Figure 1(b) and (c) respectively [5-7].

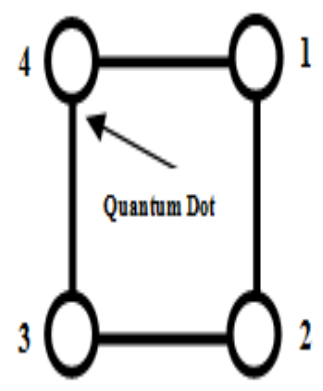

(a)

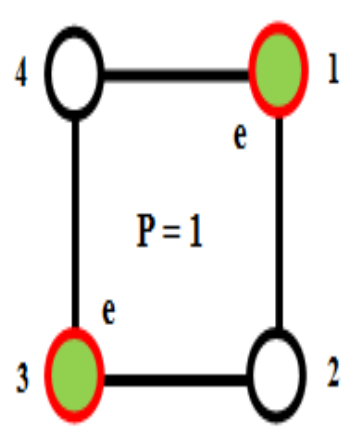

(b)

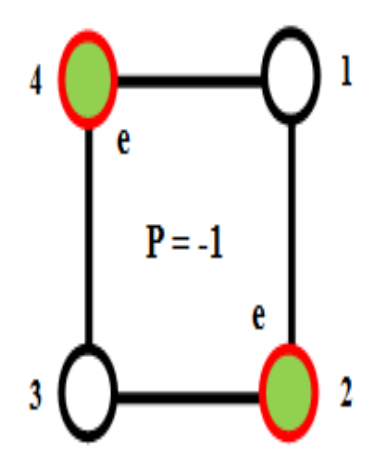

(c)

Figure 1. (a) Basic Four-dot QCA Cell, (b) Binary Logic '1' Encoding, and (c) Binary Logic ' 0 ' Encoding [3]

\subsection{QCA Elementary Nanostructures}

The Figure 2 illustrates a typical realization of binary wire in QCA technology. The polarization of the input cell in QCA wire causes the whole array to assume the same polarization due to coulombic interactions. There is no flow of current in QCA circuits only the electronic polarization changes; this results in extremely low power dissipation in QCA circuits/systems [4].

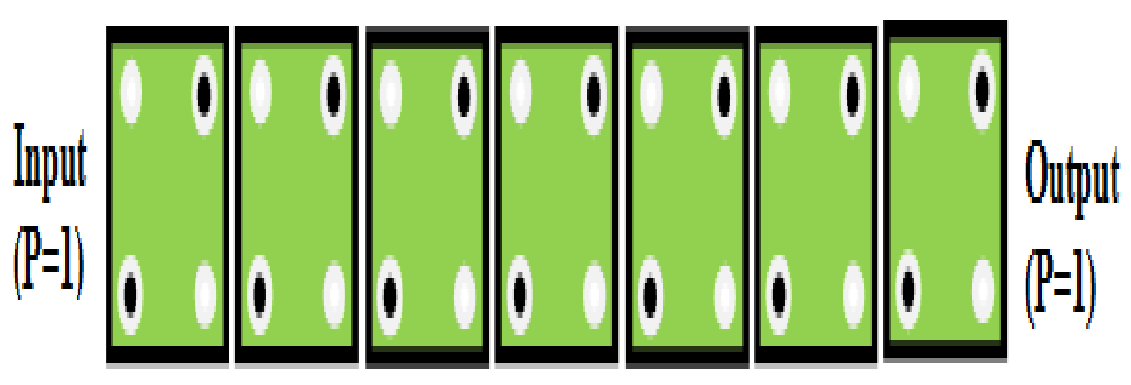

Figure 2. A Typical QCA Wire Implementation [4] 


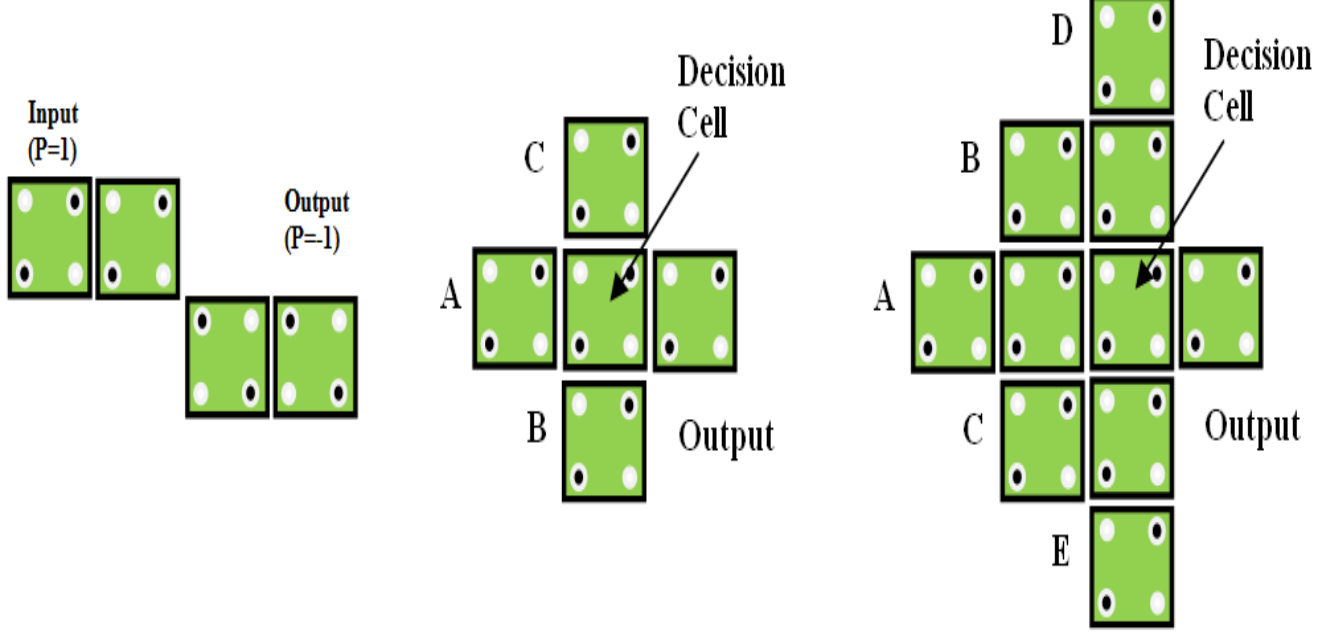

Figure 3. (a) A QCA Inverter Realization [5] (b) Basic 3-input QCA Majority Gate [5] and (c) A 5-input Majority Gate [8]

The Figure 3 (a) illustrates a mostly used QCA inverter implementation [5]. The fundamental computing gate in QCA is 3-input majority gate as depicted in Figure 3 (b). The middle cell in 3-input majority gate acquire a particular polarization as per majority of three inputs. The majority gate can be programmed to act as AND and $O R$ gates by fixing polarization of any of 3 inputs to ' -1 ' or ' 1 ' respectively. So, circuit/system designers can implement any Boolean function using majority and inverter gates [5-7] in QCA nanotechnology. The output of 3-input majority gate can be computed by equation (1).

$$
\operatorname{MAJ}(\mathrm{A}, \mathrm{B}, \mathrm{C})=\mathrm{AB}+\mathrm{BC}+\mathrm{AC}
$$

The 5-input majority gate is an emerging gate used in implementing complex QCA circuits/systems [8]. The Figure 3 (c) depicts a fundamental 5-input QCA based majority gate. The output of a 5-input QCA based majority gate can be computed using equation (2).

$\mathrm{MAJ}(\mathrm{A}, \mathrm{B}, \mathrm{C}, \mathrm{D}, \mathrm{E})=\mathrm{ABC}+\mathrm{ABD}+\mathrm{ABE}+\mathrm{ACD}+\mathrm{ACE}+\mathrm{ADE}+\mathrm{BCD}+\mathrm{BCE}+\mathrm{BDE}+\mathrm{CDE}$

\subsection{QCA Clocking}

The signal flow in QCA based circuits/systems is caused by application of appropriate clocking scheme. There are 4 different clock zones labeled as Clock-0 to Clock-3. Further, there are 4-phases in each clock zone namely Switch, Hold, Release, and Relax. There are $90^{\circ}$ of phase-shift between 4 adjacent phases in a particular clock zone. The Figure 4 illustrates 4-phase clocking scheme. The clocking causes input polarization to flow in a pipelined manner from inputs to outputs [10]. 


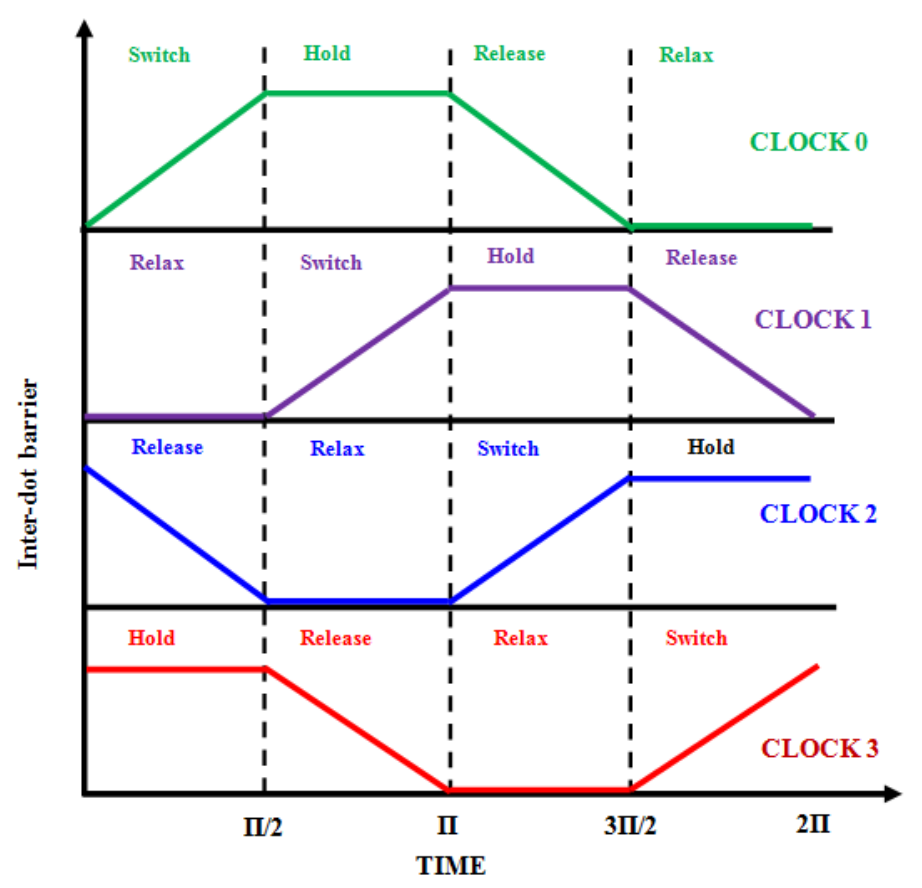

Figure 4. Clocking Flow in Different Clock Zones [10]

\section{QCA Technology Developments}

QCA nanotechnology is still under experimental research and not commercializes yet. There are experimental demonstrations of this technology by researchers in metal-island, molecular, semiconductor and magnetic QCA fabrication technologies. The first experimental demonstration of QCA technology was done in metal-island technology [1112]. The four quantum dots were fabricated using Aluminum islands and connected among themselves using Aluminum oxide tunnel junctions and capacitors. The major limitation of this realization is that the metal island need to be kept at cryogenic temperatures for having electron switching as the size of quantum dot is in microns. Semiconductor QCA implementations [13-16] have been realized in advanced CMOS processes using GaAs/AlGaAs material system. However, existing CMOS processes did not reached a level where mass fabrication of QCA devices would become a reality. However, with continued feature size scaling as the size approaches a few nanometers, the semiconductor QCA devices would become able to operate at room temperature.

Molecular QCA are attracting lot of attention nowadays as they promise room temperature operation [16-19]. The molecules perform function of QCA cells and are attached to the substrate surface at adjacent sites. The redox sites act as quantum dots. The free electrons available in the molecule switch position between redox sites due to electrostatic interactions. The critical obstacles are selection of molecules, the design of appropriate clocking mechanism and how to interface molecules to real world. Magnetic QCA [20-22] works on the principle of interaction between magnetic nanoparticles. The magnetic moment of nanoparticles is used to represent two binary states in digital circuits. The moment is either parallel or anti-parallel in reference to the axis of the chain. The Magnetic QCA devices can work at room temperature in existing fabrication technology. The major limitation of magnetic QCA is limited speed of few hundred megahertz. The applications of magnetic QCA could be explored in low power areas where speed is not importance. 


\section{Evolution of QCA Adders}

Adders are widely used in data processing and computing circuits such as Arithmetic Logic Unit (ALU) of processors. The performance of modern processors is decided by the speed, area, and power consumption of adders. Hence, design of a robust adder in QCA technology is basic necessity for a high performance arithmetic unit. Many researchers have introduced efficient and robust adder designs in QCA nanotechnology. Table 1 illustrates evolution of QCA adders over the period of time since their inception [5, 7, 2359].

Table 1. Evolution of QCA Adders

\begin{tabular}{|c|c|c|}
\hline Authors & Year & Research Brief \\
\hline Tougaw and Lent [5] & 1994 & $\begin{array}{l}\text { Demonstrated that QCA cells could be used to implement XOR gate } \\
\text { and 1-bit full adder. The full adder design uses } 5 \text { majority gates and } \\
3 \text { inverters and } 9 \text { wire crossovers. }\end{array}$ \\
\hline Lent and Tougaw [7] & 1997 & $\begin{array}{l}\text { Realized an adiabatically pipelined QCA full adder circuit using } \\
\text { new adiabatic switching paradigm allowing clocked control of QCA } \\
\text { cell arrays. }\end{array}$ \\
\hline Wang et al. [23] & 2003 & $\begin{array}{l}\text { Presented a novel QCA adder design by minimizing number of } \\
\text { majority gates, inverters and wire crossovers. }\end{array}$ \\
\hline $\begin{array}{l}\text { Rumi Zhang et al. } \\
\text { [24] }\end{array}$ & 2005 & $\begin{array}{l}\text { Proved that QCA based ripple-carry and bit-serial adders are faster } \\
\text { than QCA based carry-look-ahead and carry-select adders. }\end{array}$ \\
\hline $\begin{array}{l}\text { H. Cho and E. } \\
\text { Swartzlander [25] }\end{array}$ & 2005 & $\begin{array}{l}\text { Designed } 4,16 \text {, and } 64 \text { bit carry lookahead adders in QCA and } \\
\text { performed comparison with QCA ripple carry adders in terms of } \\
\text { complexity, area, and delay. It was observed that carry lookahead } \\
\text { adders offer modular designs. }\end{array}$ \\
\hline $\begin{array}{l}\text { H. Cho and E. } \\
\text { Swartzlander [26] }\end{array}$ & 2006 & $\begin{array}{l}\text { Discussed modular design of conditional sum adders (CSA) in QCA } \\
\text { nanotechnology with different operand sizes and compared these } \\
\text { designs with ripple carry adders and carry look-ahead adders. }\end{array}$ \\
\hline K. Kim et al. [27] & 2007 & $\begin{array}{l}\text { The failure analysis of QCA adders has been done and emphasized } \\
\text { to exploit proper clocking schemes. }\end{array}$ \\
\hline $\begin{array}{l}\text { H. Cho and E. } \\
\text { Swartzlander [28] }\end{array}$ & 2007 & $\begin{array}{l}\text { QCA based Ripple carry, carry lookahead, and conditional sum } \\
\text { adders are designed, analyzed, and compared with different operand } \\
\text { sizes in terms of area, complexity and delay. }\end{array}$ \\
\hline $\begin{array}{l}\text { T. J. Dysart and P.M. } \\
\text { Kogge [29] }\end{array}$ & 2007 & $\begin{array}{l}\text { QCA designs of adders are analyzed based on probabilistic transfer } \\
\text { matrices using triple modular redundancy (TMR). }\end{array}$ \\
\hline $\begin{array}{l}\text { E. Tabrizizadeh et al. } \\
\text { [30] }\end{array}$ & 2008 & $\begin{array}{l}\text { A new QCA serial adder has been designed which is delay- } \\
\text { insensitive QCA. The registers are used to control the flow of } \\
\text { information within the circuit modules. }\end{array}$ \\
\hline $\begin{array}{l}\text { I. Hänninen and } \mathrm{J} \text {. } \\
\text { Takala [31] }\end{array}$ & 2008 & $\begin{array}{l}\text { Performed analysis of multi-bit ripple carry, conditional sum, and } \\
\text { serial QCA adders. }\end{array}$ \\
\hline $\begin{array}{l}\text { I. Hänninen and } \mathrm{J} \text {. } \\
\text { Takala [32] }\end{array}$ & 2008 & $\begin{array}{l}\text { Probabilistic analysis of a complete QCA multi-bit ripple carry } \\
\text { adder (RCA) unit is performed and concluded that the reliability } \\
\text { depends linearly on the failure rates of the macro level components. }\end{array}$ \\
\hline $\begin{array}{l}\text { H. Cho and E. } \\
\text { Swartzlander [33] }\end{array}$ & 2009 & $\begin{array}{l}\text { A new QCA based fast and efficient carry flow adder (CFA) is } \\
\text { designed. }\end{array}$ \\
\hline
\end{tabular}




\begin{tabular}{|c|c|c|}
\hline $\begin{array}{l}\text { I. Hänninen and } \mathrm{J} \text {. } \\
\text { Takala [34] }\end{array}$ & 2010 & $\begin{array}{l}\text { The analysis of logical bits lost in standard binary conditional sum, } \\
\text { pipelined ripple carry, and carry look-ahead QCA adders have been } \\
\text { performed. }\end{array}$ \\
\hline $\begin{array}{l}\text { I. Hänninen and } \mathrm{J} \text {. } \\
\text { Takala [35] }\end{array}$ & 2010 & $\begin{array}{l}\text { A noise rejecting QCA full adder has been introduced for arithmetic } \\
\text { units. Also designs of robust serial and pipelined ripple carry adders } \\
\text { have been presented and functionally verified. }\end{array}$ \\
\hline $\begin{array}{l}\text { V. Pudi and K. } \\
\text { Sridharan [36] }\end{array}$ & 2011 & $\begin{array}{l}\text { Presented efficient QCA designs of Ladner-Fischer prefix and } \\
\text { hybrid adders. }\end{array}$ \\
\hline F. Bruschi et al. [37] & 2011 & $\begin{array}{l}\text { Proposed QCA design of adder circuits by utilizing minority gates } \\
\text { instead of majority gates resulting in significant reduction in number } \\
\text { of cells required. }\end{array}$ \\
\hline M. Gladshtein [38] & 2011 & $\begin{array}{l}\text { Presented an algorithm for addition of two operands encoded by the } \\
\text { Johnson-Mobius Code (JMC) to design a QCA serial decimal adder. } \\
\text { The new adder is compared with parallel and conventional designs } \\
\text { in terms of complexity, area, cost function and propagation delay. }\end{array}$ \\
\hline W. Liu et al. [39] & 2012 & $\begin{array}{l}\text { Reviewed several QCA adder designs and evaluated them based on } \\
\text { newly proposed QCA performance metrics. }\end{array}$ \\
\hline W. Liu et al. [40] & 2012 & $\begin{array}{l}\text { Introduced two new cost-efficient binary-coded decimal (BCD) } \\
\text { adders in QCA nanotechnology. The first adder is based on the carry } \\
\text { flow adder (CFA) and employs a conventional correction method } \\
\text { and the second adder utilizes the carry lookahead algorithm. Both } \\
\text { adders outperform previous adders in terms of delay and overall } \\
\text { cost. }\end{array}$ \\
\hline $\begin{array}{l}\text { V. Pudi and } \mathrm{K} . \\
\text { Sridharan [41] }\end{array}$ & 2012 & $\begin{array}{l}\text { Introduced new concepts for majority logic and utilized them to } \\
\text { design efficient QCA ripple carry adder (RCA) and various prefix } \\
\text { adders. The number of majority gates employed for n-bit Kogge-- } \\
\text { Stone, RCA, Ladner-Fischer, Brent-Kung and Han-Carlson adders } \\
\text { are also computed. }\end{array}$ \\
\hline S. Perri et al. [42] & 2012 & $\begin{array}{l}\text { The properties of auxiliary propagate and generates signals have } \\
\text { been explored to minimize the addition time and number of majority } \\
\text { gates required for QCA adders. Also introduced three new } \\
\text { formulations of basic logic equations used in the designs of fast } \\
\text { binary adders. }\end{array}$ \\
\hline $\begin{array}{l}\text { V. Pudi and K. } \\
\text { Sridharan [43] }\end{array}$ & 2012 & $\begin{array}{l}\text { Introduced two new decomposition theorems helpful in reducing } \\
\text { delay in multi-bit QCA adders. }\end{array}$ \\
\hline M. Gladshtein [44] & 2013 & $\begin{array}{l}\text { Demonstrated two original QCA serial decimal adder/subtractor } \\
\text { designs using a delay element implemented by short length of QCA } \\
\text { wire as a function element avoiding traditional parallel Boolean } \\
\text { logic processing. }\end{array}$ \\
\hline $\begin{array}{l}\text { A. Thamos and H. T. } \\
\text { Vergos [45] }\end{array}$ & 2013 & $\begin{array}{l}\text { Presented QCA implementations of parallel prefix Ling-Carry } \\
\text { adders that use a Ladner-Fischer parallel-prefix algorithm for carry } \\
\text { computation. }\end{array}$ \\
\hline $\begin{array}{l}\text { K. A. Escobar and R. } \\
\text { P Ribas [46] }\end{array}$ & 2013 & $\begin{array}{l}\text { Presented the design of a complex adder using the parallel prefix } \\
\text { addition algorithms. }\end{array}$ \\
\hline $\begin{array}{l}\text { Bibhash Sen et al. } \\
\text { [47] }\end{array}$ & 2013 & $\begin{array}{l}\text { A new multilayer QCA full adder design implemented using five- } \\
\text { input majority gate. The new design is analyzed by implementing } \\
\text { ripple carry adders of different operand sizes and compared with } \\
\text { other adder designs. }\end{array}$ \\
\hline
\end{tabular}




\begin{tabular}{|c|c|c|}
\hline $\begin{array}{l}\text { M. Kianpour et al. } \\
\text { [48] }\end{array}$ & 2014 & $\begin{array}{l}\text { Successfully designed, implemented and simulated a new QCA full } \\
\text { adder having minimum delay, area and complexities. }\end{array}$ \\
\hline $\begin{array}{l}\text { E. McLarnon et al. } \\
\text { [49] }\end{array}$ & 2014 & $\begin{array}{l}\text { A bit erasure analysis of carry flow, Brent-Kung and Ladner-Fischer } \\
\text { adders is presented. }\end{array}$ \\
\hline M. Gladshtein [50] & 2014 & $\begin{array}{l}\text { A new technique 'delay-based processing-in-wire' is proposed for } \\
\text { designing QCA 1-bit and multi-bit serial decimal adders based on } \\
\text { serial decimal data storage-transfer-processing and Johnson-Mobius } \\
\text { encoding. }\end{array}$ \\
\hline S. Perri et al. [51] & 2014 & $\begin{array}{l}\text { A new QCA adder is introduced which outperforms all existing } \\
\text { adders and exhibits best area-delay tradeoff. }\end{array}$ \\
\hline D. Kunalan et al. [52] & 2014 & $\begin{array}{l}\text { A QCA based 4-bit ripple carry adder is introduced using the new } \\
\text { reversible Feynman and Toffoli gates. }\end{array}$ \\
\hline $\begin{array}{l}\text { Z. Mohammadi et al. } \\
\text { [53] }\end{array}$ & 2014 & $\begin{array}{l}\text { Conventional reversible Toffoli and Fredkin gates based full adder is } \\
\text { designed and investigated. }\end{array}$ \\
\hline $\begin{array}{l}\text { M. Hayati and A. } \\
\text { Reza [54] }\end{array}$ & 2014 & $\begin{array}{l}\text { Optimized one-bit full adder, full subtractor, full adder/ full } \\
\text { subtractor and a 4-bit carry flow adder in QCA nanotechnology. } \\
\text { Also the cell-missing defects are investigated for defect } \\
\text { characterization and testing of the proposed full adder. }\end{array}$ \\
\hline D. Abedi et al. [55] & 2015 & $\begin{array}{l}\text { The new QCA adder has been designed using non-adjacent clock } \\
\text { zones (shifted by } 180^{\circ} \text { ) for the two crossing wires. This results in } \\
\text { reduction of QCA cell count and area consumption. }\end{array}$ \\
\hline R. Farazkish [56] & 2015 & $\begin{array}{l}\text { A novel QCA fault-tolerant full-adder has been proposed which is } \\
\text { significantly more robust to single or multi-faults in missing cells, } \\
\text { misalignment cells and dislocation cells. }\end{array}$ \\
\hline A. Roohi et al. [57] & 2015 & $\begin{array}{l}\text { Introduced an efficient layered fault-tolerant design for 1-bit full } \\
\text { adder. }\end{array}$ \\
\hline $\begin{array}{l}\text { S. Hashemi and } \mathrm{K} . \\
\text { Navi [58] }\end{array}$ & 2015 & $\begin{array}{l}\text { Introduced a novel robust QCA full-adder implemented using an } \\
\text { efficient five-input majority gate. It is then used to realize ripple } \\
\text { carry adders with different operand sizes. }\end{array}$ \\
\hline $\begin{array}{l}\text { A. N. Bahar and S. } \\
\text { Waheed [59] }\end{array}$ & 2016 & $\begin{array}{l}\text { Proposed a new flexible 5-input majority gate and a new efficient } \\
\text { QCA based full-adder design. The new design offers significant } \\
\text { improvement in comparison to the previous designs in terms of } \\
\text { covered area and number of QCA cells. }\end{array}$ \\
\hline G. Singh et al. [62] & 2016 & $\begin{array}{l}\text { Introduced a new novel robust XOR gate in QCA technology which } \\
\text { can be employed in arithmetic and communication circuits/systems. }\end{array}$ \\
\hline G. Singh et al. [63] & 2017 & $\begin{array}{l}\text { Presented design and performance analysis of a new efficient } \\
\text { coplanar quantum-dot cellular automata adder and also carried out } \\
\text { its energy dissipation analysis. }\end{array}$ \\
\hline
\end{tabular}

\section{QCA Full Adder Designs}

A full adder has 3 inputs and two outputs 'Sum' and ' $C_{\text {out }}$ '. The ' $A$ ', ' $B$ ', and ' $C_{i n}$ ' are inputs as shown in Figure 5. The generalized Boolean equations for 'Sum' and ' $C_{\text {out }}$ ' are given in equations (3) and (4) respectively:

$$
\begin{gathered}
\mathrm{Sum}=(\mathrm{A}) \operatorname{xor}(\mathrm{B}) \operatorname{xor}\left(\mathrm{C}_{\mathrm{in}}\right) \\
\mathrm{C}_{\text {out }}=(\mathrm{A}) \text { and }(\mathrm{B}) \operatorname{or}(\mathrm{B}) \text { and }\left(\mathrm{C}_{\text {in }}\right) \operatorname{or}\left(\mathrm{C}_{\mathrm{in}}\right) \text { and }(\mathrm{A})
\end{gathered}
$$




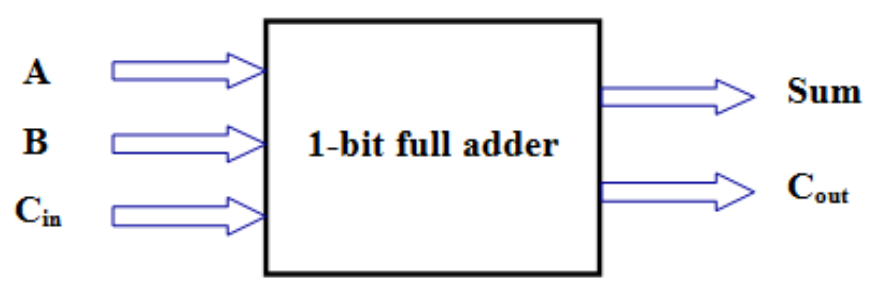

Figure 5. Block Diagram of 1-bit Full Adder

QCA adders are classified mainly into two major categories depending on the type of wire crossover method used in the design:

a) Coplanar Adders: All QCA cells in design are arranged in only one layer and employs normal and rotated cells $\left(45^{\circ}\right)$ for wire crossover or no wire crossover at all. However, in coplanar wire crossover is prone to thermal defects. So, it should be better if there is no wire-crossover in designed QCA circuits as it enhances reliability of the circuit. Clock zone based crossover is the most recent, robust, fast, and low cost coplanar wire crossover technique. It basically uses two clock zones separated by 180 degree to cross two wires. Delay optimization become difficult in this technique as the complexity of the circuit increases.

b) Multilayer Adders: It employs multiple layers of cells similar to many metal layers used in integrated circuits (ICs). The major advantages are effective crossing of signals and lesser area required than coplanar QCA circuits. However, it still lacks physical implementation and moreover the fabrication cost is expected to increase by threefold.

\subsection{Coplanar Full Adders}

\section{Tougaw and Lent Adder}

The first design for a 1-bit QCA full adder was proposed by Tougaw and Lent [5]. It uses 5 majority gates and 3 inverters. The 'Sum' and ' $C_{\text {out }}$ ' outputs are calculated in terms of majority gates and inversion operation as described by equations (5) and (6) below:

$$
\begin{gathered}
\operatorname{Sum}=\operatorname{M3}\left(M 3\left(\bar{A}, B, C_{\text {in }}\right), M 3\left(A, \bar{B}, C_{\text {in }}\right), M 3\left(A, B, \bar{C}_{\text {in }}\right)\right. \\
C_{\text {out }}=M 3\left(A, B, C_{\text {in }}\right)
\end{gathered}
$$

The number of majority logic gates, inverters and wire crossovers required are $5 n, 3 n$ and $9 n$ for an n-bit Tougaw and Lent full adder. The delay is $n+1 / 4$ clock cycles for $n$-bit adder. The major limitation of this adder is high number of wire crossovers and poor clocking scheme. Also it uses $45^{\circ}$ rotated QCA cell for wire crossovers which provide poor coupling. The Figure 6 (a) shows the schematic diagram of 1-bit Tougaw and Lent full adder.

\section{Wang Adder}

Wang used majority logic reduction methods to optimize and simplify the layout of Tougaw and Lent full adder [23]. The n-bit Wang adder comprises of only $3 n$ majority gates, $2 n$ inverters and $6 n$ wire crossovers. The complexity is reduced; however, the propagation delay is equal to Tougaw and Lent adder. The delay of n-bit Wang adder $n$ $+1 / 4$ clock cycles. The output carry computation is similar to Tougaw adder. The 'Sum' is calculated in terms of majority gates and inversion operation as described by equation (7).

$$
\operatorname{Sum}=M 3\left(\bar{C}_{\text {out }}, C_{\text {in }}, M 3\left(A, B, \bar{C}_{\text {in }}\right)\right.
$$


The major advantage of this adder is efficient clocking scheme which minimizes crosstalk between inputs. The Figure 6 (b) shows the schematic diagram of 1-bit Wang full adder.

\section{Abedi Adder}

This QCA adder design employs clocking mechanism for wire crossovers [55]. It uses two non-adjacent clock zones shifted by $180^{\circ}$ for crossing two wires. This results in the design of QCA full adder with significant reduction in QCA cell count and area. This adder structure uses $3 n$ majority gates, $2 n$ inverters and no wire crossover for an n-bit adder. The 'Sum' function for this adder is computed using equation (8) below.

$$
\text { Sum }=M 3\left(\bar{C}_{\text {out }}, M 3\left(A, B, \bar{C}_{\text {in }}\right), \bar{C}_{\text {in }}\right)
$$

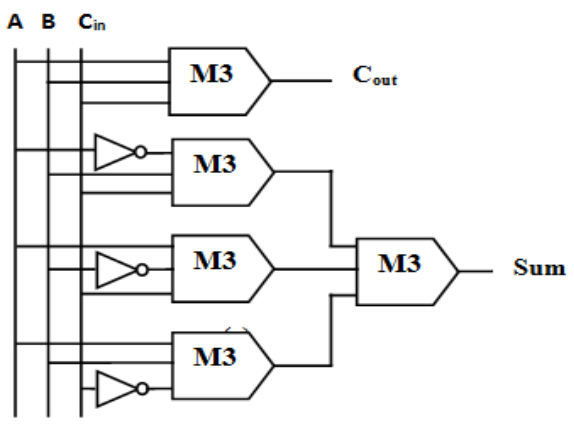

(a)

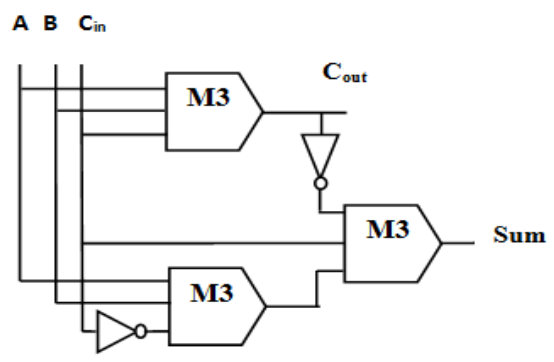

(b)

Figure 6. Schematic Diagrams of (a) 1-bit Tougaw and Lent Full Adder [5] and (b) 1-bit Wang Full Adder [23]

\section{Hanninen and Takala Adder}

This QCA adder is optimized logical layout of Wang's adder [31]. The optimization has been performed by efficiently rearranging the clocking zones to realize a robust adder design. It uses only $3 n, 2 n$, and $3 n$ number of majority gates, inverters and wire crossovers for an n-bit Hanninen and Takala adder. However, the delay of this adder has degraded which is $n+l$ clock cycles for an n-bit adder.

\section{R. Azghadi adder}

This QCA adder design employs 5-input majority gate along with a 3-input majority gate and an inverter to realize a 1-bit full adder [60]. The use of 5-input majority gate facilitates simplifying complex logical functions and achieve improved results. This also helps in reducing gate counts required to implement digital functions. The Figure 7 shows the schematic diagram of 1-bit QCA Azghadi full adder.

\section{A $B \quad C_{\text {in }}$}

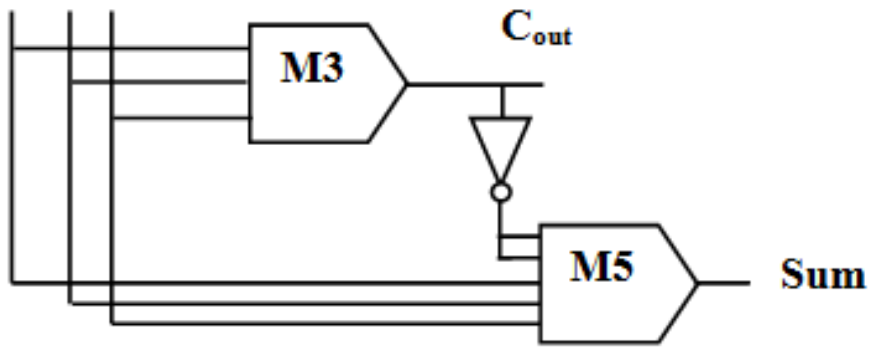

Figure 7. Schematic of 1-bit QCA Azghadi Full Adder [60] 


\subsection{Multi-Layer Adders}

\section{Zhang Adder}

This QCA adder is just multi-layer implementation of Wang's adder. It utilizes the same addition algorithm [24]. It uses $3 n$ majority gates, $2 n$ inverters and $3 n$ multilayer wire crossovers for an n-bit adder. The delay is $n$ clock cycle which is less than Wang's adder. The cost of coplanar and multi-layer crossings is quite different.

\section{Cho Adder}

A new optimized carry flow adder was introduced by Cho [25]. The path from carry in to carry out make use of one majority gate. This adder uses only one clocking zone delay per bit which considerably minimizes delay for large-size adders. It uses $3 n$ majority gates, $2 n$ inverters and $2 n$ crossovers respectively. The delay of an n-bit Cho adder is $\frac{n+2}{4}$.

\section{Pudi Adder}

Pudi proposed QCA based Brent-Kung adder design [36]. It is basically a parallel prefix adder. Majority logic reduction techniques have been used to improve performance in terms of delay for large-bit adders. This adder structure minimizes carry computation to a prefix computation. The output 'Sum' and ' $C_{\text {out }}$ ' of Pudi's adder are calculated by equations (9) and (10) respectively.

$$
\begin{gathered}
\operatorname{Sum}=M 3\left(\bar{C}_{\text {out }}, M 3\left(g, p, \bar{C}_{\text {out }}\right), C_{\text {in }}\right) \\
C_{\text {out }}=M 3\left(g, p, C_{\text {in }}\right)
\end{gathered}
$$

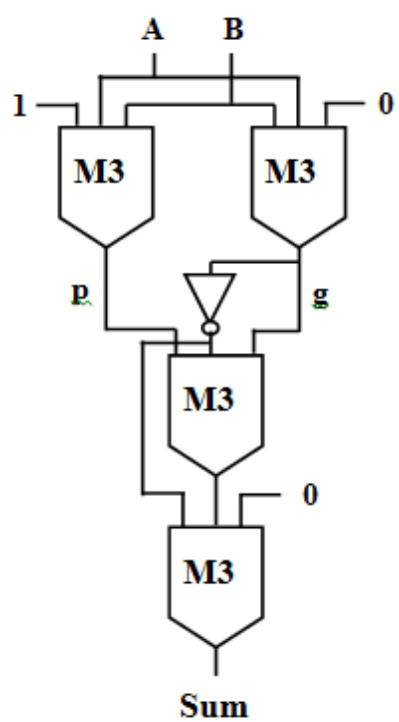

Figure 8. Schematic Diagram of 1-bit Pudi Adder [36]

Where, $g=A B$ and $p=A+B$

The schematic of a 1-bit Pudi adder is shown in Figure 8. An n-bit Pudi adder requires ، $8 n-3 \log _{2}(n)-4$, majority gates, ' $n$ ' inverters and ' $n\left(\log _{2} n-3\right)+\log _{2} n+3 n+3$, wire crossovers. The delay of an n-bit Pudi adder is $\frac{2 \log _{2} n+3}{4}+\frac{n\left(\log _{2} n-2\right)}{32}$. 


\section{Qanbari and Nadooshan Adder}

The output carry is computed by a three-input majority gate and then the carry is inverted and is given as input to two inputs of the five-input majority gate [61]. The other three inputs of five-input majority gate are $A, B$, and $C_{\text {in }}$ respectively. The output of the five-input majority gate generates the 'Sum' output. The output 'Sum' and ' $C_{\text {out }}$ ' is calculated by equations (11).

$$
\operatorname{Sum}=\operatorname{M5}\left(A, B, C_{\text {in }}, \bar{C}_{\text {out }}, \bar{C}_{\text {out }}\right)
$$

\section{Bibhash Sen Adder}

This multilayer adder employs one five-input majority gate, one three-input majority gate and computes the 'Sum' output in just two clock zones [47]. The output 'Sum' is computed by equation (11). The output carry $C_{\text {out }}$ is computed according to equation (6) using a three-input majority gate in the first layer and is directly forwarded to the output. It just needs one clock zone. Then, the $C_{\text {out }}$ is transmitted upwards by placing a cell in the second layer. This output carry signal is finally fed into the five-input majority gate in the third layer. Inputs $A, B$, and $C_{i n}$ are applied in the first layer, propagated to third layer by vertically stacked cells and supplied as input to the five-input majority gate. The fiveinput majority gate generates the output Sum.

\section{Performance Metrics of QCA Adders}

QCA nanotechnology has been emerging as a prospective candidate to replace CMOS technology in future digital circuits. The most of the performance metrics of QCA circuits are mapped from CMOS technology and used to evaluate QCA circuits. Being a new technology, there is a need to investigate the suitable performance metrics for this nanotechnology. W. Liu et al. [39] proposed new metrics for QCA circuits and also formulated new cost functions for optimization of QCA circuits. They found that the major metrics are- number of majority/inverter gates, propagation delay, and number and type of wire crossovers. Hence, these performance metrics and cost functions are considered to compare QCA circuits.

\section{Number of majority/inverter gates}

A typical QCA circuit comprises of three-input majority gates and inverters. Both of these are main computing gates in QCA technology, any digital circuit can be realized from these. So, the numbers of majority and inverter gates basically affect complexity and area of a QCA circuit. Also, these days five-input majority gates are also used to realize complex digital functions and to reduce the number of levels of three-input majority gates required.

\section{Number and type of wire crossovers}

In QCA circuits, wire crossovers play very important role in deciding complexity and area computations of QCA circuits. The crossing of two wires carrying two different signals is a critical issue in QCA nanotechnology. There are two types of wire crossovers in QCA circuits- coplanar crossings and multi-layer wire crossings. In coplanar wire crossing QCA cells are arranged in one layer only and require high precision in alignment. In multi-layer wire crossovers, QCA cells are arranged in multiple-layers. The fabrication cost of this kind of wire crossover is high as compared to coplanar wire crossover as minimum atleast three layers are needed. Also accurate distance between two vertical cells is required for achieving desired kink energy. The equation (12) is generally used to approximate cost of multi-layer crossing [39]:

$$
\operatorname{Cost}_{M L}=n \times \operatorname{Cost}_{C P}
$$


Where, $\operatorname{Cost}_{C P}$ is the cost of a coplanar wire crossover, Cost $_{M L}$ is the cost of a multilayer wire crossover and ' $n$ ' is multiplying factor. The value of ' $n$ ' is typically assumed 3 as multi-layer wire crossovers use at least three different layer. The wire crossovers impose implementation constraints in designing complex QCA circuits. The number of wire crossovers should be minimum.

\section{Propagation Delay (Latency)}

It is most important index of speed of a QCA circuit. The number of clock zones is measure of propagation delay. A clock zone is $1 / 4^{\text {th }}$ of a clock period. Lesser the number of clock zones required in a QCA circuit, better is the speed. The number of clocking zones used in a QCA circuit is a measure of delay or latency.

A generalized QCA cost function [39] based on the number of majority gates (M) and inverters (I), number and type of wire crossover $(\mathrm{C})$, and the propagation delay $(\mathrm{T})$ as described by equation (13):

$$
\operatorname{Cost}_{Q C A}=\left(M^{k}+I+C^{l}\right) \times T^{p} \quad 1 \leq k, l, p
$$

Table 2. Comparison of $\mathbf{n}$-bit Coplanar QCA Full Adders

\begin{tabular}{|c|c|c|c|c|c|c|}
\hline $\begin{array}{l}\text { Coplanar } \\
\text { Adders }\end{array}$ & $\begin{array}{c}\text { Basic } \\
\text { Full Adder } \\
\text { Structure }\end{array}$ & $\begin{array}{c}\text { Type } \\
\text { and No. of } \\
\text { Majority } \\
\text { Gates used }\end{array}$ & $\begin{array}{r}\text { No. of } \\
\text { Inverters }\end{array}$ & $\begin{array}{c}\text { No. of } \\
\text { wire- } \\
\text { crossovers }\end{array}$ & $\begin{array}{l}\text { Dela } \\
\text { y } \\
(\mathrm{Cyc} \\
\text { les })\end{array}$ & $\begin{array}{r}\text { No. } \\
\text { of QCA } \\
\text { Cells }\end{array}$ \\
\hline $\begin{array}{c}\text { Tougaw and } \\
\text { Lent } \\
{[5]}\end{array}$ & $\begin{array}{l}\text { Tougaw } \\
\text { and Lent }\end{array}$ & $\begin{array}{l}\text { Three- } \\
\text { input }=5 n\end{array}$ & $3 n$ & $9 n$ & $\begin{array}{c}\mathrm{n}+ \\
1 / 4\end{array}$ & 192 \\
\hline Wang [23] & Wang & $\begin{array}{l}\text { Three- } \\
\text { input }=3 n\end{array}$ & $2 n$ & $6 n$ & $\begin{array}{c}n+ \\
1 / 4\end{array}$ & 145 \\
\hline $\begin{array}{c}\text { Hanninen } \\
\text { and Takala } \\
\text { [31] }\end{array}$ & Wang & $\begin{array}{r}\text { Three- } \\
\text { input }=3 n\end{array}$ & $2 n$ & $3 n$ & $\mathrm{n}+1$ & 102 \\
\hline $\begin{array}{l}\text { D. Abedi } \\
\text { [55] }\end{array}$ & Wang & $\begin{array}{c}\text { Three- } \\
\text { input }=3 n\end{array}$ & $2 n$ & $\begin{array}{c}\text { Not } \\
\text { Required }\end{array}$ & $\mathrm{n}$ & 59 \\
\hline $\begin{array}{l}\text { S. Hashemi } \\
\text { and K. Navi } \\
{[58]}\end{array}$ & $\begin{array}{r}\text { M. R. } \\
\text { Azghadi }\end{array}$ & $\begin{array}{c}\text { Three- } \\
\text { input }=1 \mathrm{n} \\
\text { Five } \\
\text { Input }=1 \mathrm{n}\end{array}$ & $2 n$ & $\begin{array}{c}\text { Not } \\
\text { Required }\end{array}$ & $\begin{array}{c}\mathrm{n}+ \\
1 / 4\end{array}$ & 71 \\
\hline $\begin{array}{c}\text { M. R. } \\
\text { Azghadi [60] }\end{array}$ & $\begin{array}{r}\text { M. R. } \\
\text { Azghadi }\end{array}$ & $\begin{array}{c}\text { Three- } \\
\text { input }=1 \mathrm{n} \\
\text { Five } \\
\text { Input }=1 \mathrm{n}\end{array}$ & $1 \mathrm{n}$ & $\begin{array}{c}\text { Not } \\
\text { Required }\end{array}$ & $\begin{array}{c}\mathrm{n}+ \\
1 / 2\end{array}$ & NS* \\
\hline
\end{tabular}

NS*- Not Specified 
Table 3. Comparison of $\mathbf{n}$-bit Multilayer QCA Full Adders

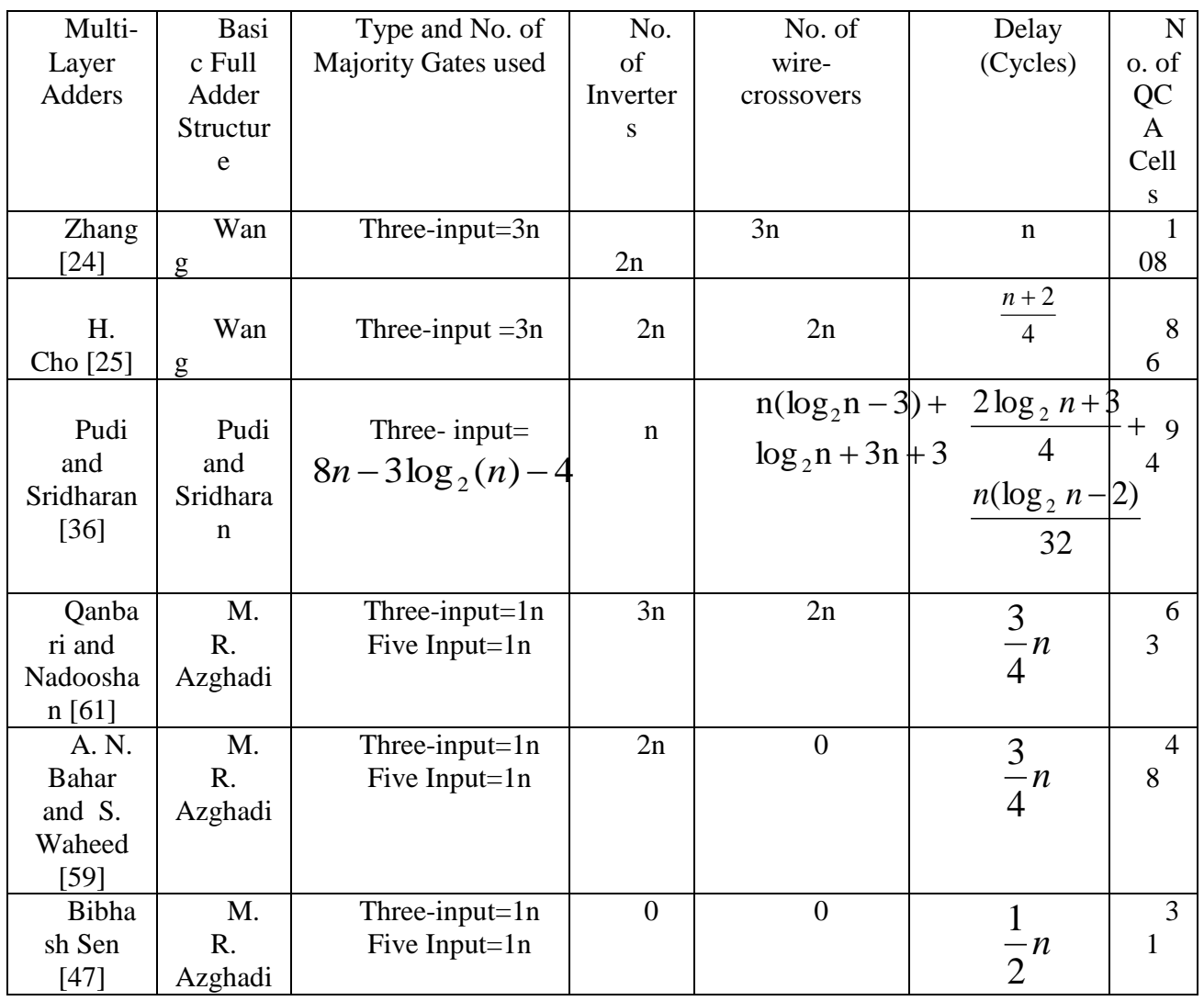

Where, $k, l, p$ are the exponential weightings for number of majority gates, wire crossovers count and propagation delay in equation (13) respectively. A QCA circuit can be optimized for different performance metrics according to weightings assigned to $\mathrm{k}, 1$, and $\mathrm{p}$.

The Table 2 shows comparison of n-bit coplanar QCA full adders and Table 3 shows comparison of n-bit multilayer QCA full adders reported in literature. The comparison has been carried out in terms of number of majority/inverter gates, type and number of wirecrossovers, propagation delay, and QCA cells used in QCA layout of various adders.

\section{Cost Function Computations for QCA Adders}

The cost functions values have been calculated for 1-bit coplanar and multilayer QCA full adders using QCA cost function described by equation (13). The coplanar QCA full adder structures considered for cost function computations are Taugaw and Lent, Wang, Hänninen, D. Abedi, S. Hashemi and K. Navi, and M. R. R. Azghadi. The cost function has been computed for multilayer QCA full adder structures like Zhang, H. Cho, Pudi and Sridharan, Qanbari and Nadooshan, Qanbari and Nadooshan, A. N. Bahar and S. Waheed , and B. Sen respectively. The cost function has been computed for QCA adder structures by giving higher priority to complexity. As complexity of a QCA circuit is decided by number of majority gates and number and type of wire crossovers, higher priority has been given to $\mathrm{k}=2$ and $\mathrm{l}=2$. The cost of a multilayer wire crossover has been taken three times of a coplanar wire crossover. From computations, we observed that Taugaw and Lent adder is most complex from complexity point of view while D. Abedi adder is least complex. Also S. Hashemi and K. Navi, and M. R. R. Azghadi adder are less complex coplanar adder structures. Similarly, from multilayer adders Pudi and Sridharan adder is most complex while B. Sen adder is least complex. The five-input majority gate is considered equivalent to two three-input majority gates as it has 10 QCA cells and also 5 
number of wires are required for it. The Figure 9 and 10 depicts the bar-graph comparison of cost function computations performed for 1-bit coplanar and multilayer QCA adders.

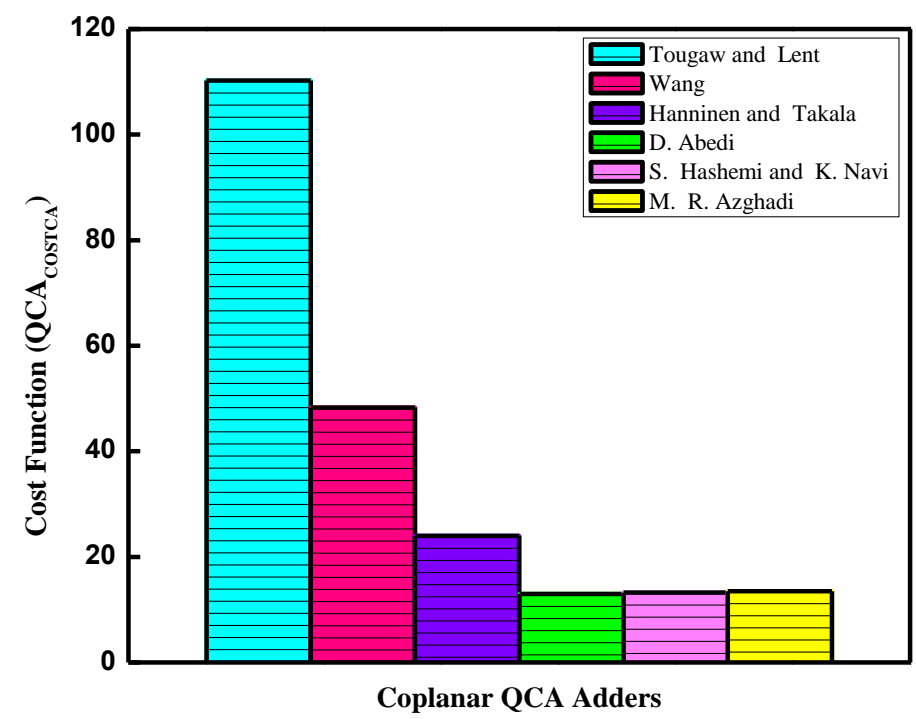

Figure 9. Cost Function for Coplanar QCA Adders

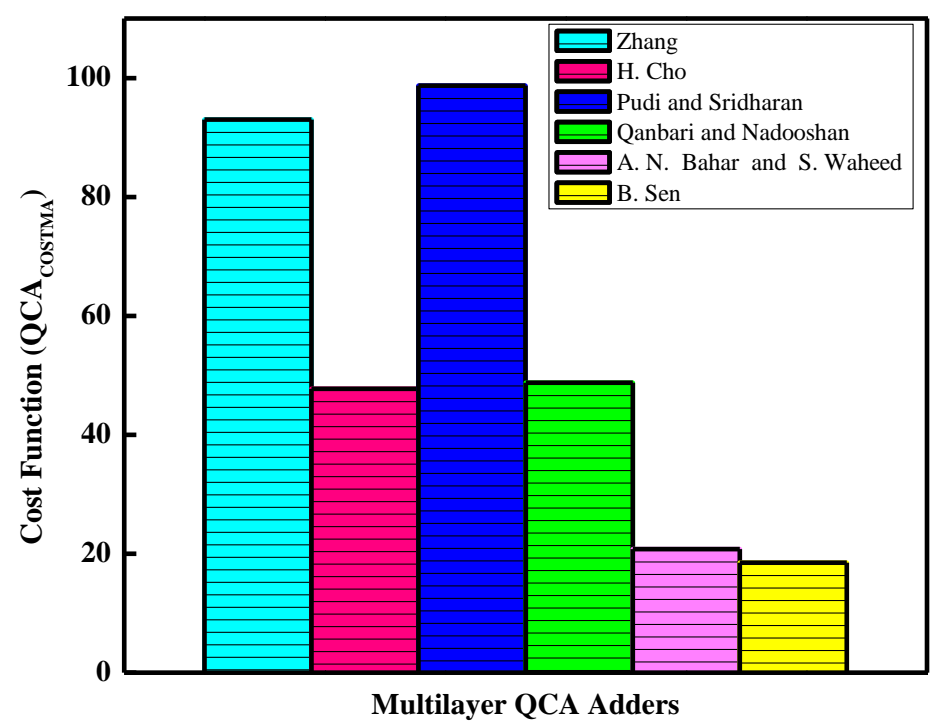

Figure 10. Cost Function for Multilayer QCA Adders

\section{Conclusion}

The continuous scaling down of feature size has pushed CMOS technology to approach its practical and theoretical limits. Lot of research efforts at nanoscale are in progress to explore alternate viable technologies for future integrated circuits. QCA is emerging as a potential technology that could be used in future computing circuits/systems replacing existing Silicon technology. This paper presents an introduction to QCA technology, QCA fundamentals, QCA technology developments, evolution of QCA adders, QCA full adder designs, performance metrics, and cost function computations for coplanar and multilayer QCA 1-bit full adders. The QCA adder designs have been compared in terms of adder type (coplanar or multilayer), basic full adder structure type, type and number of majority gates used, number of inverters, number of wire-crossovers, delay, and number 
of QCA cells. Also, the cost functions have been computed for both coplanar and multilayer QCA adders by giving higher priority to complexity.

\section{References}

[1] Emerging Research Devices (ERD), International Technology Roadmap for Semiconductors (ITRS), 2013 Edition, http://www.itrs.net/Links/2013ITRS/Home2013.html

[2] G. Bourianoff, "The Future of Nanocomputing", IEEE Computer Society, (2003) August.

[3] C. S. Lent, P. D. Taugaw, W. Porod and G. H. Berstein, "Quantum Cellular Automata", Nanotechnology (IOPScience), vol. 4, no. 1, (1993).

[4] C. S. Lent and Tougaw P. D., "Lines of Interacting Quantum-Dot Cells: A Binary Wire", Journal of Applied Physics, vol. 74, (1993), pp. 6227-6233.

[5] P. D. Taugaw and C. S. Lent, "Logical Devices Implemented Using Quantum Cellular Automata", Journal of Applied Physics, vol. 75, (1994), pp. 1818-1825.

[6] W. Porod, C. S. Lent., G. H. Bernstein, A. O. Orlov, I. Amlani, G. L. Snider and J. L. Merz, "QuantumDot Cellular Automata: Computing With Coupled Quantum Dots", International Journal of Electronics [Taylor and Francis], vol. 86, no. 5, (1999), pp. 549-590.

[7] C. S. Lent and P. D. Tougaw, "A Device Architecture for Computing with Quantum Dots", Proceedings of the IEEE, vol. 85, (1997), pp. 541-557.

[8] K. Navi, S. Sayedsalehi, R. Farazkish and M. Rahimi Azghadi, "Five-Input Majority Gate, a New Device For Quantum-Dot Cellular Automata", Journal of Computational \& Theoretical Nanoscienence (ASP), vol. 7, no. 8, (2010), pp. 1546-1553.

[9] J. Timler and C. S. Lent, "Power gain and dissipation in quantum-dot cellular automata", Journal of Applied Physics, vol. 91, no. 2, (2002), pp. 823-831.

[10] V. Vankamamidi, M. Ottavi and F. Lombardi, "Two-Dimensional Schemes for Clocking/Timing of QCA Circuits", IEEE Transactionson Computer-Aided Design of Integrated Circuits and Systems, vol. 27, no. 1, (2008), pp. 34-44.

[11] A. O. Orlov, I. Amlani, G. H. Bernstein, C. S. Lent and G. L. Snider, "Realization of a functional cell for quantum-dot cellular automata, Science Magazine, vol. 227, (1997), pp. 928-930.

[12] I. Amlani, A. O. Orlov, R. K. Kummamuru, G. H. Bernstein, C. S. Lent and G. L. Snider, "Experimental demonstration of a leadless quantum-dot cellular automata cell", Applied Physics Letters, vol. 77, no. 5, (2000), pp. 738-740.

[13] S. Gardelis, C. G. Smith, J. Cooper, D. A. Ritchie, E. Linfield and Y. Jin, "Evidence for Transfer of Polarization in a Quantum-Dot Cellular Automata Cell Consisting of Semiconductor Quantum Dots", Physical Review B, vol. 67, no. 3, (2003), pp. 1-3.

[14] F. Perez-Martinez, I. Farrer, D. Anderson, G. Jones, D. A. Ritchie, S. Chorley, and C. G. Smith, "Demonstration of a Quantum Cellular Automata Cell in a GaAs/AlGaAs Heterostructures", Applied Physics Letters, vol. 91, 032102, (2007), pp. 1-3.

[15] C. G. Smith, S. Gardelis, A. Rushforth, R. Crook, J. Cooper, D. A. Ritchie, E. Linfield, Y. Jin, and M. Pepper, "Realization of Quantum-Dot Cellular Automata Using Semiconductor Quantum Dots", Superlattices and Microstructures, vol. 34, Issues 3-6, (2003), pp. 195-203.

[16] M. Mitic, M. C. Cassidy, K. Petersson, R. Starrett, E. Gauja, R. Brenner, R. Clark, A. Dzurak, C. Yang, and D. Jamieson, "Demonstration of a Silicon-Based Quantum Cellular Automata Cell", Applied Physics Letters, vol. 89, 013503, (2006), pp. 1-3.

[17] K. Hennessy and C. S. Lent, "Clocking of Molecular Quantum-Dot Cellular Automata, "Journal of Vacuum Science \& Technology”, vol. B19, no. 5, (2001), pp. 1752-1755.

[18] E. P. Blair and C. S. Lent, "An Architecture for Molecular Computing Using Quantum-dot Cellular Automata", Proceedings of 3rd IEEE Conference on Nanotechnology, (2003), pp. 402-405.

[19] R. A. Joyce, H. Qi, T. P. Fehlner, C. S. Lent, A. O. Orlov and G. L. Snider, "A System to Demonstrate the Bistability in Molecules for Application in a Molecular QCA Cell", IEEE Nanotechnology Materials and Devices Conference, (2009) June 2-5, pp. 46-49.

[20] R. P. Cowburn and M. E. Welland, "Room Temperature Magnetic Quantum Cellular Automata", Science Magazine, vol. 287, (2000), pp. 1466-1468.

[21] A. Imre, G. Csaba, G. H. Bernstein, W. Porod and V. Metlushko, "Investigation of Antiferromagnetic Ordering Along Chains of Coupled Nanomagnets", Proceedings of 3rd IEEE Conference on Nanotechnology, vol. 2, (2003), pp. 20-23.

[22] B. Lambson, D. Carlton and J. Bokor, "Exploring the Thermodynamic Limits of Computation in Integrated Systems: Magnetic Memory, Nanomagnetic Logic, and the Landauer limit", Physical Review Letters, vol. 107, 010604, (2011), pp. 1-4.

[23] W. Wang, K. Walus and G. A. Jullien, "Quantum-Dot Cellular Automata Adders", in proceedings of Proceedings of third IEEE Conference on Nanotechnology, (2003), pp. 461-464.

[24] R. Zhang, K. Walus, W. Wei and G. A. Jullien, "Performance comparison of quantum-dot cellular automata adders", Proceedings of IEEE International Symposium on Circuits and Systems (ISCAS), vol. 3, (2005), pp. 2522-2526. 
[25] H. Cho and E. E. Swartzlander, "Pipelined Carry Lookahead Adder Design in Quantum-dot Cellular Automata", Proceedings of Conference Record of the Thirty-Ninth Asilomar Conference on Signals, Systems and Computers, (2005), pp. 1191-1195.

[26] H. Cho and E. Swartzlander, "Modular Design of Conditional Sum Adders using Quantum-dot Cellular Automata", Proceedings of Sixth IEEE Conference on Nanotechnology (IEEE-NANO), (2006), pp. 363366.

[27] K. Kim, K. Wu and R. Karri, "The Robust QCA Adder Designs Using Composable QCA Building Blocks", IEEE Trans. on Computer Aided Design of Integrated Circuits and Systems, vol. 26, no. 1, (2007) January, pp-176-183.

[28] H. Cho and E. Swartzlander, "Adder designs and Analyses for Quantum-Dot Cellular Automata", IEEE Trans. on Nanotechnology, vol. 6, no. 3, (2007) May, pp. 374-383.

[29] T. J. Dysart and P. M. Kogge, "Probabilistic Analysis of a Molecular Quantum-Dot Cellular Automata Adder", Proceedings of 22nd IEEE International Symposium on Defect and Fault Tolerance in VLSI Systems, (2007), pp. 478-486.

[30] E. Tabrizizadeh, H. Mohaqeq and A. Vafaei, "Designing QCA Delay-Insensitive Serial Adder", Proceedings of First International Conference on Emerging Trends in Engineering and Technology (ICETET), (2008), pp. 447-452.

[31] I. Hänninen and J. Takala, "Arithmetic Design on Quantum-Dot Cellular Automata Nanotechnology" Springer-Verlag Berlin Heidelberg, SAMOS 2008, LNCS 5114, pp. 43-52.

[32] I. Hänninen and J. Takala, "Reliability of n-Bit Nanotechnology Adder", Proceedings of IEEE Computer Society Annual Symposium on VLSI, (2008), pp. 34-39.

[33] H. Cho and E. Swartzlander, "Adder and Multiplier Design in Quantum -Dot Cellular Automata", IEEE Transactions on Computers, vol. 58, no. 6, (2009), pp. 721-727.

[34] I. Hänninen and J. Takala, "Irreversible Bit Erasures in Binary Adders", Proceedings of $10^{\text {th }}$ IEEE Conference on Nanotechnology (IEEE-NANO), (2010), pp. 223-226.

[35] I. Hänninen and J. Takala, "Binary Adders on Quantum-Dot Cellular Automata", Springer, J Sign Process Syst, vol. 58, (2010), pp. 87-103.

[36] Pudi and Sridharan, "Efficient Design of a Hybrid Adder in Quantum-Dot Cellular Automata", IEEE Transactions On Very Large Scale Integration (VLSI) Systems, vol. 19, no. 9, (2011), pp. 1535-1548.

[37] F. Bruschi, F. Perini, V. Rana and D. Sciuto, "An Efficient Quantum-Dot Cellular Automata Adder", Proceedings of Design, Automation \& Test in Europe Conference \& Exhibition (DATE), (2011), pp. 14.

[38] M. Gladshtein, "Quantum-Dot Cellular Automata Serial Decimal Adder", IEEE Transactions on Nanotechnology, vol. 10, no. 6, (2011), pp. 1377-1382.

[39] W. Liu, L. Lu, M. O’Neill and E. Swartzlander, "A First Step Toward Cost Functions for Quantum-Dot Cellular Automata Designs", IEEE Transactions on Nanotechnology, vol. 13, no. 3, (2014), pp. 476-487.

[40] W. Liu, L. Lu, M. O'Neill and E. Swartzlander, "Cost-efficient decimal adder design in Quantum-dot cellular automata", Proceedings of IEEE International Symposium on Circuits and Systems (ISCAS), (2012), pp. 1347-1350.

[41] V. Pudi and K. Sridharan, "Low Complexity Design of Ripple Carry and Brent-Kung Adders in QCA", IEEE Transactions on Nanotechnology, vol. 11, no. 1, (2012), pp. 105-119.

[42] S. Perri and P. Corsonello, "New Methodology for the Design of Efficient Binary Addition Circuits in QCA", IEEE Transactions on Nanotechnology, vol. 11, no. 6, (2012), pp. 1192-1200.

[43] V. Pudi and K. Sridharan, "New Decomposition Theorems on Majority Logic for Low-Delay Adder Designs in Quantum Dot Cellular Automata", IEEE Transactions on Circuits and Systems-II: Express Briefs, vol. 59, no. 10, (2012), pp. 678-682.

[44] M. Gladshtein, "Design and simulation of novel adder / subtractors on quantum-dot cellular automata", Microelectronics Journal (Elsevier), vol. 44, (2013), pp. 545-552.

[45] A. Thamos and H. T. Vergos, "Fast parallel-prefix Ling-carry adders in QCA nanotechnology", Proceedings of IEEE 20th International Conference on Electronics, Circuits, and Systems (ICECS), (2013), pp. 565-568.

[46] K. A. Escobar and R. P Ribas, "Parallel prefix adder design using quantum-dot cellular automata", Proceedings of 26th Symposium on Integrated Circuits and Systems Design (SBCCI), (2013), pp. 1-6.

[47] B. Sen, A. Rajoria and B. K. Sikdar, "Design of Efficient Full Adder in Quantum-Dot Cellular Automata", The ScientificWorld Journal (Hindawi), vol. 2013, Article ID 250802, 10 pages.

[48] M. Kianpour, R. Sabbaghi-Nadooshan and K. Navi, "A Novel Design of 8-Bit Adder/Subtractor by Quantum Dot Cellular Automata", Journal of Computer and System Sciences (Elsevier), (2014), vol. 80, pp. 1404-1414.

[49] E. McLarnon, M. O' Neill, W. Liu and I. Hänninen, "Bit Erasure Analysis of Binary Adders in Quantum-dot Cellular Automata", Proceedings of the 14th IEEE International Conference on Nanotechnology Toronto, Canada, (2014), August 18-21.

[50] M. Gladshtein, "Delay-Based Processing-in-Wire for Design of QCA Serial Decimal Arithmetic Units", ACM Journal on Emerging Technologies in Computing Systems, vol. 10, no. 2, Article 13, (2014) February. 
[51] S. Perri, P. Corsonello and G. Cocorullo, "Area-Delay Efficient Binary Adders in QCA", IEEE Transactions on Very Large Scale Integration (VLSI) Systems, vol. 22, no. 5, (2014), pp. 1174-1179.

[52] D. Kunalan, C. L. Cheong, C. F. Chau and A. B. Ghazali, "Design of a 4-bit Adder using reversible logic in Quantum-Dot Cellular Automata (QCA)", Proceedings of IEEE International Conference on semiconductor electronics (ICSE), (2014), pp. 60-63.

[53] Z. Mohammadi and M. Mohammadi, "Implementing a one-bit reversible full adder using quantum-dot cellular automata”, Quantum Information Processing (Springer), vol. 13, no. 2, (2014), pp. 2127-2147.

[54] M. Hayati and A. Rezaei, "Design of Novel Efficient Adder and Subtractor for Quantum-Dot Cellular Automata", International Journal of Circuit Theory and Applications, vol. 43, no. 10, pp. 1446-1454, (2014) October.

[55] D. Abedi, G. Jaberipur and M. Sangsefidi, "Coplanar Full Adder in Quantum-Dot Cellular Automata via Clock-Zone Based Crossover", IEEE Transactions on Nanotechnology, vol. 14, no. 3, (2015), pp. 497504.

[56] R. Farazkish, "A New Quantum-Dot Cellular Automata Fault-Tolerant Full-Adder", Journal of Computational Electronics (Springer), vol. 14, Issue 2, (2015) June, pp. 506-514.

[57] A. Roohi, R. F. DeMara and N. Khoshavi, "Design and evaluation of an ultra-area-efficient faulttolerant QCA full adder", Microelectronics Journal, vol. 46, (2015), pp. 531-542.

[58] S. Hashemi and K. Navi, "A Novel Robust QCA Full-adder”, Proceedings of 5th International Biennial Conference on Ultrafine Grained and Nanostructured Materials (UFGNSM15), (2015), pp. 376-380.

[59] A. N. Bahar and S. Waheed, "Design and Implementation of an Efficient Single Layer Five Input Majority Voter Gate in Quantum-Dot Cellular Automata", SpringerPlus, 2016; 5:636. DOI: 10.1186/s40064-016-2220-7.

[60] M. Rahimi Azghadi, O. Kavehie and K. Navi, "A Novel Design for Quantum-dot Cellular Automata Cells and Full Adders", Journal of Applied Sciences, vol. 7, no. 22, (2007), pp. 3460-3468.

[61] M. Qanbari and R. Sabbaghi-Nadooshan, "Two Novel Quantum-Dot Cellular Automata Full Adders", Journal of Engineering, vol. 2013, Article ID 561651, 6 pages.

[62] G. Singh, R. K. Sarin and B. Raj, "A Novel Robust Exclusive-or Function Implementation in QCA nanotechnology With Energy Dissipation Analysis", Journal of Computational Electronics, vol. 15, issue 2, (2016), pp. 455-465.

[63] G. Singh, B. Raj and R. K. Sarin, "Design and performance analysis of a new efficient coplanar quantum-dot cellular automata adder", Indian journal of Pure \& Applied Physics, vol. 55, (2017) February, pp. 97-103. 
International Journal of Hybrid Information Technology

Vol. 10, No.4 (2017) 